zu erreichen. Bei manchen Patienten nach Angaben der European Society of Hypertension Task Force immerhin $15-20 \%$ - sind sogar drei Medikamente erforderlich.

\section{Fixkombis für Kombi-Therapie verfügbar}

Hochdruckexperten raten bei schwerer Hypertonie, hohem kardiovaskulären Risiko und deutlicher Diskrepanz zwischen Ausgangs- und Zielwert dazu, von Anfang an eine Zweierkombination $\mathrm{zu}$ erwägen. Für eine solche Initialtherapie ist die niedrig dosierte Fixkombi aus Perindopril und dem Thiazid-Analogon Indapamid erhältlich (Preterax ${ }^{\oplus} \mathrm{N}$ und normal dosiert als BiPreterax ${ }^{\star} \mathrm{N}$ ). Werte unter $140 / 90 \mathrm{mmHg}$ sind das Ziel bis ins hohe Alter. Auch bei über 80-Jährigen gibt es weniger Infarkte und Insulte, wie die Studie HYVET mit Perindopril/ Indapamid bestätigt.

Argumente für die Differenzialtherapie nach der Anfangstherapie liefern die Studien ASCOT und ACCOMPLISH. In beiden hat sich eine Kombination RASBlocker/Kalzium-Antagonist (Perindopril bzw. Benazepril/Amlodipin) bewährt. Mit Valsartan/Amlodipin (Exforge ${ }^{\varpi}$, Dafiro ${ }^{\circledast}$ ), Olmesartan/Amlodipin (Sevikar ${ }^{\circledast}$, Vocado $^{\varpi}$ ) sowie Telmisartan/ Amlodipin (Twynsta $^{\circledR}$ ) gibt es Fixkombinationen aus solchen Wirkstoffklassen. Zudem wird etwa Enalapril/Nitrendipin (Eneas ${ }^{\varpi}$ ) oder Enalapril/Lercanidipin (Zanipress ${ }^{\circledR}, \mathrm{Za}-$ neril $^{\triangleright}$ ) eingesetzt. Falls nötig, kommt ein Diuretikum dazu, etwa in den Fixkombinationen Exforge $\mathrm{HCT}^{\circledast}$, Sevikar $\mathrm{HCT}^{\circledast}$ oder Vocado HCT ${ }^{\circledast}$.

(Robert Bublak)

Ärzte Zeitung, 01.07.2013
ÜBRIGENS...

... ist Bluthochdruck nicht besonders sexy. In einer italienischen Studie zeigten sich Hypertonikerinnen deutlich unzufriedener mit ihrem Liebesleben als normotone Frauen. Die Zufriedenheit stieg allerdings wieder, wenn der Hochdruck behandelt wurde.

... sollte an beiden Armen der Blutdruck gemessen werden. Systolische Unterschiede von $10 \mathrm{mmHg}$ oder mehr weisen auf eine erhöhte Morbidität und Mortalität hin.

... steigt der Druck mit jedem Körnchen Salz. Sinkt der Salzkonsum von 9-12 g auf 5-6 g, fällt der systolische Wert um $4 \mathrm{mmHg}$, der diastolische Wert um $2 \mathrm{mmHg}$.

\title{
Blutdruck im Schlaf senken - geht das?
}

Schlafmangel zieht viele ungünstige metabolische Effekte nach sich. Und auch der Blutdruck steigt. Ausreichend lange zu schlafen könnte daher helfen, die Werte zu drücken.

Eine zu kurze Schlafdauer hat Auswirkungen auf den Stoffwechsel, die ihrerseits zur Pathogenese der Hypertonie beitragen können. Studien haben ergeben, dass Schlafentzug den Appetit steigert, die Insulinsensitivität beeinträchtigt und den LDL-Cholesterinspiegel erhöht. Führt der Weg vom zu kurzen Schlaf zur Hypertonie also über Adipositas, Diabetes und Hypercholesterinämie? Und hilft es, länger zu schlafen, um die Druckwerte zu senken?

\section{Um 20\% erhöhtes Hypertonierisiko bei fünf Stunden Schlaf}

Mediziner der New Yorker Columbia University haben versucht, diese Fragen zu beantworten. Sie bedienten sich dabei der Daten von Teilnehmerinnen der Nurses' Health Study I/II. An den beiden Untersuchungen waren insgesamt mehr als 150.000 Frauen beteiligt gewesen. Unab- hängig vom Alter und nach Risikofaktoren abgeglichen, war bei Frauen, die höchstens fünf Stunden am Tag schliefen, die Hypertonieprävalenz um knapp 20\% erhöht. Als Vergleichsgruppe dienten Frauen, die länger als sieben Stunden schliefen. Anders sah es bei der Inzidenz aus. Ein um 11\% höheres Risiko, neu an Bluthochdruck zu erkranken, zeigte sich nur bei Kurzschläferinnen, die noch keine 50 Jahre alt waren.

Um herauszufinden, auf welche Weise der Kurzschlaf den Druck erhöht, berechneten die Forscher den Einfluss von Diabetes, Hypercholesterinämie und Body-Mass-Index (BMI). Als relevantes Bindeglied trat dabei lediglich der BMI in Erscheinung. Kurzer Schlaf scheint hungrig, träge und dick zu machen. Umgekehrt könnte daher ein verlängerter Schlaf vor Hypertonie schützen, indem er regelmäßigen Sport und ein gesünderes Essverhalten ermöglicht - und auf diese Weise den Körper schlank sowie den Blutdruck tief hält.

(rb)

Am J Hypertens 2013, online 4. April www.springermedizin.de/ Sommerakademie CME

In der e.Akademie von Springer Medizin können Sie bis zum 28. August 2013 kostenfrei punkten.

Zum Thema "Hypertonie" finden Sie unter www.springermedizin.de/sommerakademie den Kurs „Resistente Hypertonie: Neue Therapieoptionen".

Um teilnehmen zu können, geben Sie bitte die Fortbildungs-Identifikationsnummer (FIN) in das entsprechende Feld ein.

\section{FIN: SOAK13uU}

kostenlos teilnehmen bis 28.08.2013

Viel Erfolg beim Punktesammeln!

Mit rund 1000 Kursen - viele davon als multimediale E-Learning-Formate - ist die e.Akademie Deutschlands erste Adresse für Online-CME-Fortbildung.

Die e.Akademie ist ein Modul von e.Med, dem Zugang zu allen digitalen Inhalten von Springer Medizin.

Testen Sie die e.Akademie 30 Tage lang kostenlos mit e.Med: www.springermedizin.de/eMed. 